\title{
Emptiness Check of Powerset Büchi Automata using Inclusion Tests
}

\author{
Souheib Baarir Alexandre Duret-Lutz \\ LIP6-CNRS, \\ Université Pierre et Marie Curie (Paris 6), \\ France \\ E-mail:Souheib.Baarir@lip6.fr,Alexandre.Duret-Lutz@lip6.fr
}

\begin{abstract}
We introduce two emptiness checks for Büchi automata whose states represent sets that may include each other. The first is equivalent to a traditional emptiness check but uses inclusion tests to direct the on-the-fly construction of the automaton. The second is impressively faster but may return false negatives. We illustrate and benchmark the improvement on a symmetry-based reduction.
\end{abstract}

\section{Introduction}

The automata-theoretic approach to modelchecking [15] uses automata on infinite words to represent a system as well as a property to check on it. Both automata are synchronized, and the resulting product automaton is examined by an emptiness check.

One drawback of this approach, known as the state explosion problem, lies in the large size of the automaton that represents the behavior of the system, and hence in the resulting size of the product automaton that has to be checked for emptiness. Several techniques have been proposed to reduce the size of both automata [14]. Some of these, like unfolding graphs [4], observation graphs [9], or symmetrybased reductions [8], share a common property: instead of an automaton $\mathcal{A}$, they build an automaton $\mathcal{B}$ whose state are a symbolic representation of a set of states of $\mathcal{A}$.

To take an example, the method of partial symmetries introduced by Haddad et al. [8] works by partitioning the set of successors $s_{1}, s_{2}, \ldots, s_{n}$ of a state of the original automaton, and using these partitions as the states $S_{1}, S_{2}, \ldots, S_{m}$ of the reduced automaton $\mathcal{B}$. However, because this might be done locally in each state, there is no guarantee that a state $s$ of the original automaton may be represented by only one state $S$ in $\mathcal{B}$. This reduced automaton can then be checked for emptiness (using any mainstream emptiness check algorithm $[11,5,7])$ with the same result as if the original automaton $\mathcal{A}$ had been checked (of course the idea is that $\mathcal{B}$ is constructed directly from the system and the property, in such a way that we avoid the construction of $\mathcal{A}$ ).

Unfortunately, these methods may well construct a "reduced" automaton that has more states that the original automaton! Indeed, if we call $\mathcal{A}$ the original automaton and $\mathcal{Q}_{\mathcal{A}}$ its set of states, this technique constructs an automaton $\mathcal{B}$ where $\mathcal{Q}_{\mathcal{B}} \subseteq 2^{\mathcal{Q}_{\mathcal{A}}}$ and may have at worst $2^{\left|\mathcal{Q}_{\mathcal{A}}\right|}$ states. However in many practical cases we still have $\left|\mathcal{Q}_{\mathcal{B}}\right|<\left|\mathcal{Q}_{\mathcal{A}}\right|$. For instance, Fig. 1 (ignore $f$ and $g$ for the moment) shows two automata $\mathcal{A}$ and $\mathcal{B}$, where $\mathcal{B}$ 's states are sets of states of $\mathcal{A}$. In the sequel, we will always use lowercase letters like $s_{i}$ to denote states of the original automaton, and uppercase letters like $S_{i}$ to denote states of the reduced automaton (which are sets of states).

Furthermore, we can see that some states of $\mathcal{A}$ may appear in several states of $\mathcal{B}$, and most importantly a state of $\mathcal{B}$ may even be a subset of another state of $\mathcal{B}$ (e.g., $S_{4} \subseteq S_{1}$ and $S_{5} \subseteq S_{2}$ ). These inclusions motivate this paper.

When the state space is constructed on-the-fly during the emptiness check, we will show that we can perform inclusion tests to reduce the number of constructed states. For instance, in Fig. 1 we can avoid constructing $S_{4}$ and $S_{5}$ because the information they represent is already included in $S_{1}$ and $S_{2}$.

To rid ourselves of the construction technique used to build $\mathcal{B}$, we present our emptiness check algorithms in a general framework of powerset automata.

Section 2 defines these automata formally, and proposes a set of 5 properties tying $\mathcal{A}$ to $\mathcal{B}$ that are sufficient to ensure that both automata are equivalent with respect to their emptiness. Section 3 presents our emptiness check algorithm for such automata. Basically such an algorithm may answer "empty" or "not empty". Section 4 shows a small modification that leads to a faster algorithm that can answer "empty" or "I don't know". The definitions and algorithms presented in the aforementioned sections are abstract in the sense that they do not presume how $\mathcal{B}$ was constructed from $\mathcal{A}$ : as an illustration, Section 5 adapts the 

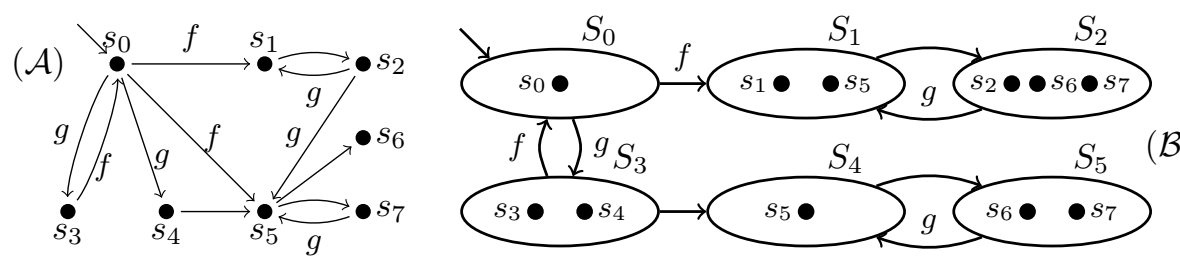

Figure 1. An example of powerset automaton $\mathcal{B}$ for an automaton $\mathcal{A}$, with $\mathcal{F}_{\mathcal{A}}=\mathcal{F}_{\mathcal{B}}=\{f, g\}$.

technique of Haddad et al. [8] to show how to construct a $\mathcal{B}$ using (partial-)symmetries, and we prove that this construction satisfies the requirements of our algorithm. We also benchmark this construction and show that although theoretically the algorithm may still explore $2^{\left|\mathcal{Q}_{\mathcal{A}}\right|}$ states in the worst case, in practice it improves the state space size by a good factor.

\section{Definitions}

We start with the definition of the automata we manipulate. We use structures that look like Generalized Büchi Automata, but without atomic propositions and with acceptance conditions on transitions. In the automata-theoretic approach, atomic propositions are only used for the computation of the synchronized product and can be ignored after this operation: the emptiness check algorithm does not need them. Putting acceptance conditions on transitions rather than on states is motivated by the fact that it is more generic: state-based acceptance conditions can be converted to transition-based acceptance conditions without adding states or transitions, while the converse is not true.

Definition 1 (UTGBA). An Unlabelled Transition-based Generalized Büchi Automaton (UTGBA) is a Büchi automaton without any atomic propositions, but with generalized acceptance conditions on transitions. It is a tuple $\mathcal{A}=\left\langle\mathcal{Q}_{\mathcal{A}}, \mathcal{Q}_{\mathcal{A}}^{0}, \mathcal{F}_{\mathcal{A}}, \Delta_{\mathcal{A}}\right\rangle$ where

- $\mathcal{Q}_{\mathcal{A}}$ is a finite set of elements called states,

- $\mathcal{Q}_{\mathcal{A}}^{0} \subseteq \mathcal{Q}_{\mathcal{A}}$ is a set of initial states,

- $\mathcal{F}_{\mathcal{A}}$ is a finite set of elements called acceptance conditions,

- $\Delta_{\mathcal{A}} \subseteq \mathcal{Q}_{\mathcal{A}} \times 2^{\mathcal{F}_{\mathcal{A}}} \times \mathcal{Q}_{\mathcal{A}}$ is the transition relation, where each transition carries a (possibly empty) set of acceptance conditions of $\mathcal{F}_{\mathcal{A}}$.

Definition 2 (Reachable states). Let $\mathcal{A}=$ $\left\langle\mathcal{Q}_{\mathcal{A}}, \mathcal{Q}_{\mathcal{A}}^{0}, \mathcal{F}_{\mathcal{A}}, \Delta_{\mathcal{A}}\right\rangle$ be a UTGBA. A state $s$ is reachable if $s \in \mathcal{Q}_{\mathcal{A}}^{0}$ or if there exists a finite sequence $\left\langle s_{0}, F_{0}, s_{1}\right\rangle\left\langle s_{1}, F_{1}, s_{2}\right\rangle \cdots\left\langle s_{n-1}, F_{n-1}, s_{n}\right\rangle$ of transitions of $\Delta_{\mathcal{A}}$, starting at an initial state $s_{0} \in \mathcal{Q}_{\mathcal{A}}^{0}$, and ending on state $s_{n}=s$. We denote $\operatorname{Reach}(\mathcal{A})$ the set of all reachable states of $\mathcal{A}$.
Definition 3 (Run \& accepting run). Let $\mathcal{A}$ be a UTGBA as above. $A$ run of $\mathcal{A}$ is an infinite sequence $\left\langle s_{0}, F_{0}, s_{1}\right\rangle\left\langle s_{1}, F_{1}, s_{2}\right\rangle \cdots$ of transitions of $\Delta_{\mathcal{A}}$, starting at an initial state $s_{0} \in \mathcal{Q}_{\mathcal{A}}^{0}$. A run is accepting if $\forall f \in$ $\mathcal{F}_{\mathcal{A}}, \forall i \geqslant 0, \exists j \geqslant i$, such that $f \in F_{j}$, i.e., if its transitions are labelled by each acceptance condition infinitely often.

We write $\operatorname{Run}(\mathcal{A})$ and $\operatorname{Acc}(\mathcal{A})$ the set of all runs and the set of all accepting runs of $\mathcal{A}$.

For $\sigma=\sigma(0) \sigma(1) \sigma(2) \cdots \in \operatorname{Run}(\mathcal{A})$, we denote $\sigma_{\text {in }}(i)$, $\sigma_{\text {acc }}(i)$, and $\sigma_{\text {out }}(i)$ the source, the acceptance condition, and the destination of the $i^{\text {th }}$ transition of $\sigma$, in other words $\sigma(i)=\left\langle\sigma_{\text {in }}(i), \sigma_{\text {acc }}(i), \sigma_{\text {out }}(i)\right\rangle$. Finally we denote $\sigma^{i}$ the suffix of $\sigma$ starting after the $i^{\text {th }}$ transition, that is: $\sigma^{i}=$ $\sigma(i) \sigma(i+1) \sigma(i+2) \cdots$.

As an example, consider $\mathcal{B}$ in Fig. 1. The acceptance conditions are $f, g$, and thus $\mathcal{B}$ has only one accepting run: $\operatorname{Acc}(\mathcal{B})=\left\{S_{0} S_{3} S_{0} S_{3} S_{0} S_{3} \ldots\right\}$.

An emptiness check tells whether Acc is empty, and here we are interested in an equivalence relation between automata that is solely based on the result of this operation.

Definition 4 (Emptiness-equivalence). Two UTGBAs, $\mathcal{A}$ and $\mathcal{B}$, are $\emptyset$-equivalent iff either both automata have an accepting run, or none have.

$$
\mathcal{A} \stackrel{\emptyset}{\equiv} \mathcal{B} \quad \text { iff } \quad \operatorname{Acc}(\mathcal{A})=\emptyset \Longleftrightarrow \operatorname{Acc}(\mathcal{B})=\emptyset
$$

Now we propose a set of 5 properties that link two UTGBA $\mathcal{A}$ and $\mathcal{B}$ such that the states of $\mathcal{B}$ are sets of states of $\mathcal{A}$, and $\mathcal{B}$ is $\emptyset$-equivalent to $\mathcal{A}$. The idea is that if we know a method to construct a $\mathcal{B}$ that verifies these sufficient conditions, we can run the emptiness check on $\mathcal{B}$ and avoid constructing $\mathcal{A}$. These properties hold in Fig. 1.

Definition 5 (६-UTGBA). Let $\mathcal{A}=\left\langle\mathcal{Q}_{\mathcal{A}}, \mathcal{Q}_{\mathcal{A}}^{0}, \mathcal{F}_{\mathcal{A}}, \Delta_{\mathcal{A}}\right\rangle$ and $\mathcal{B}=\left\langle\mathcal{Q}_{\mathcal{B}}, \mathcal{Q}_{\mathcal{B}}^{0}, \mathcal{F}_{\mathcal{B}}, \Delta_{\mathcal{B}}\right\rangle$ be two UTGBAs. $\mathcal{B}$ is a $\wp$ UTGBA (powerset UTGBA) over $\mathcal{A}$ if it satisfies the following properties:

$$
\begin{aligned}
\mathcal{Q}_{\mathcal{B}} & \subseteq 2^{\mathcal{Q}_{\mathcal{A}}} \backslash\{\emptyset\} \\
\mathcal{F}_{\mathcal{B}} & =\mathcal{F}_{\mathcal{A}} \\
\bigcup_{S \in \mathcal{Q}_{\mathcal{B}}^{0}} S & =\mathcal{Q}_{\mathcal{A}}^{0}
\end{aligned}
$$




$$
\begin{aligned}
& \forall\left\langle s, F, s^{\prime}\right\rangle \in \Delta_{\mathcal{A}}, \forall S \in \operatorname{Reach}(\mathcal{B}), \\
& \quad s \in S \Longrightarrow \exists S^{\prime} \in \mathcal{Q}_{\mathcal{B}} \text { s.t. } s^{\prime} \in S^{\prime}, \text { and }\left\langle S, F, S^{\prime}\right\rangle \in \Delta_{\mathcal{B}}
\end{aligned}
$$

$\forall\left\langle S, F, S^{\prime}\right\rangle \in \Delta_{\mathcal{B}}, \forall s^{\prime} \in S^{\prime}, \exists s \in S$, s.t. $\left\langle s, F, s^{\prime}\right\rangle \in \Delta_{\mathcal{A}}$

Proposition 1. Let $\mathcal{A}=\left\langle\mathcal{Q}_{\mathcal{A}}, \mathcal{Q}_{\mathcal{A}}^{0}, \mathcal{F}_{\mathcal{A}}, \Delta_{\mathcal{A}}\right\rangle$ and $\mathcal{B}=$ $\left\langle\mathcal{Q}_{\mathcal{B}}, \mathcal{Q}_{\mathcal{B}}^{0}, \mathcal{F}_{\mathcal{B}}, \Delta_{\mathcal{B}}\right\rangle$ be two UTGBAs such that $\mathcal{B}$ is a $\wp-$ UTGBA over $\mathcal{A}$. Then $\mathcal{A} \stackrel{\emptyset}{\equiv} \mathcal{B}$.

Proof. We want to show that $\exists \sigma \in \operatorname{Acc}(\mathcal{A}) \Longleftrightarrow \exists \sigma^{\prime} \in$ $\operatorname{Acc}(\mathcal{B})$.

$(\Longrightarrow)$ Let $\sigma=\left\langle s_{0}, F_{0}, s_{1}\right\rangle\left\langle s_{1}, F_{1}, s_{2}\right\rangle \cdots \in \operatorname{Acc}(\mathcal{A})$. Since $s_{0} \in \mathcal{Q}_{\mathcal{A}}^{0}$ we can use (3) and find an $S_{0} \in \mathcal{Q}_{\mathcal{B}}^{0}$ such that $s_{0} \in S_{0}$. Since $S_{0}$ is reachable in $\mathcal{B}$ and contains $s_{0}$, we can use (4) to find an $S_{1} \in \mathcal{Q}_{\mathcal{B}}$ such that $s_{1} \in S_{1}$ and $\left\langle S_{0}, F_{0}, S_{1}\right\rangle \in \Delta_{\mathcal{B}}$. Likewise, because $S_{1}$ is reachable in $\mathcal{B}$ and contains $s_{1}$ by construction, we can use (4) again to find an $S_{2} \in \mathcal{Q}_{\mathcal{B}}$ such that $s_{2} \in S_{1}$ and $\left\langle S_{1}, F_{1}, S_{2}\right\rangle \in \Delta_{\mathcal{B}}$. Iterating (4) we can construct a sequence $\sigma^{\prime}=\left\langle S_{0}, F_{0}, S_{1}\right\rangle\left\langle S_{1}, F_{1}, S_{2}\right\rangle \cdots \in \operatorname{Run}(\mathcal{B})$ such that $s_{i} \in S_{i}$ for all $i$. Since $\mathcal{F}_{\mathcal{B}}=\mathcal{F}_{\mathcal{A}}(2)$ and $\sigma^{\prime}$ visits each acceptance condition as often as $\sigma, \sigma^{\prime} \in \operatorname{Acc}(\mathcal{B})$.

$(\Longleftarrow)$ Let $\sigma^{\prime}=\left\langle S_{0}, F_{0}, S_{1}\right\rangle\left\langle S_{1}, F_{1}, S_{2}\right\rangle \cdots \in \operatorname{Acc}(\mathcal{B})$. Let's build a tree whose nodes (except the root) are states of $\mathcal{A}$. Let's call $\perp$ the root of the tree at depth 0 . The nodes of depth $n>0$ are exactly the states in $S_{n-1}$. The father $s$ of any node $s^{\prime}$ at depth $n>1$ is chosen among the nodes of depth $n-1$ such that $\left\langle s, F_{n-1}, s^{\prime}\right\rangle \in \Delta_{\mathcal{A}}$; (5) guarantees that such a node $s$ exists. The father of any node at depth 1 is $\perp$. All edges of this tree, except those leaving the root node, correspond to transitions of $\Delta_{\mathcal{A}}$.

The set of nodes at depth $n>0$ is a subset of $\mathcal{Q}_{\mathcal{A}}$, which is finite, so although this tree is infinite it has a finite degree. By König's lemma it contains an infinite branch. The sequence constructed by following the edges of this infinite branch and ignoring the first edge (leaving $\perp)\left\langle s_{0}, F_{0}, s_{1}\right\rangle\left\langle s_{1}, F_{1}, s_{2}\right\rangle \cdots$ is an accepting run of $\mathcal{A}$. Indeed it is a run of $\mathcal{A}\left(s_{0} \in \mathcal{Q}_{\mathcal{A}}^{0}\right)$ that visits each acceptance condition as often as $\sigma^{\prime}$.

We now develop two propositions that introduce the emptiness check algorithm. (For brevity, we omit the proofs of these propositions and the following, and refer the interested reader to our technical report [1].) Both propositions use the following notation.

Definition 6 (Substitution of initial states). Let $\mathcal{A}=$ $\left\langle\mathcal{Q}, \mathcal{Q}^{0}, \mathcal{F}, \Delta\right\rangle$ be a UTGBA, and $T \subseteq \mathcal{Q}$ a set of states of $\mathcal{A}$. We denote $\mathcal{A}[T]$ the automaton sharing the same structure as $\mathcal{A}$ but using the set $T$ as initial states. In other words $\mathcal{A}[T]=\langle\mathcal{Q}, T, \mathcal{F}, \Delta\rangle$.
The next proposition can be observed on Fig. 1: since no run that traverses state $S_{1}$ is accepting, then neither are the runs that traverse state $S_{4}$ because $S_{4} \subseteq S_{1}$.

Proposition 2. Let $\mathcal{B}=\left\langle\mathcal{Q}_{\mathcal{B}}, \mathcal{Q}_{\mathcal{B}}^{0}, \mathcal{F}, \Delta_{\mathcal{B}}\right\rangle$ be a $\wp-U T G B A$ over $\mathcal{A}=\left\langle\mathcal{Q}_{\mathcal{A}}, \mathcal{Q}_{\mathcal{A}}^{0}, \mathcal{F}, \Delta_{\mathcal{A}},\right\rangle$ and consider two states $T$ and $D$ of $\mathcal{Q}_{\mathcal{B}}$ such that $D \subseteq T$. We have

$$
\operatorname{Acc}(\mathcal{B}[\{T\}])=\emptyset \Longrightarrow \operatorname{Acc}(\mathcal{B}[\{D\}])=\emptyset
$$

The following proposition allows us to split a transition $\langle R, F, T\rangle$ into a set of transitions $\left\langle R, F, T_{1}\right\rangle, \ldots,\left\langle R, F, T_{n}\right\rangle$ with $T_{1} \cup \cdots \cup T_{n}=T$, while preserving $\emptyset$-equivalence. Doing so might require adding new states and transitions to the automaton. Basically we want to substitute $T$ by an automaton $\mathcal{C}$ that has $T_{1}, \ldots, T_{n}$ as initial states, and that is $\emptyset$-equivalent to $\mathcal{A}[T]$. Fig. 2 illustrates this proposition. It will prove useful to apply such a decomposition if some $T_{i}$ states have already been visited.

Proposition 3 (Decomposition of a transition in a $\wp$-UTGBA). Let $\mathcal{B}=\left\langle\mathcal{Q}_{\mathcal{B}}, \mathcal{Q}_{\mathcal{B}}^{0}, \mathcal{F}, \Delta_{\mathcal{B}}\right\rangle$ be a $\wp-U T G B A$ over $\mathcal{A}=\left\langle\mathcal{Q}_{\mathcal{A}}, \mathcal{Q}_{\mathcal{A}}^{0}, \mathcal{F}, \Delta_{\mathcal{A}}\right\rangle$. Consider a transition $\langle R, F, T\rangle \in \Delta_{\mathcal{B}}$ and let $\mathcal{C}=\left\langle\mathcal{Q}_{\mathcal{C}}, \mathcal{Q}_{\mathcal{C}}^{0}, \mathcal{F}, \Delta_{\mathcal{C}}\right\rangle$ be a $\wp$-UTGBA over $\mathcal{A}[T]$. The automaton $\mathcal{B}^{\prime}=\left\langle\mathcal{Q}_{\mathcal{B}} \cup\right.$ $\left.\mathcal{Q}_{\mathcal{C}}, \mathcal{Q}_{\mathcal{B}^{\prime}}^{0}, \mathcal{F}, \Delta_{\mathcal{B}^{\prime}}\right\rangle$ where

$\mathcal{Q}_{\mathcal{B}^{\prime}}^{0}= \begin{cases}\left(\mathcal{Q}_{\mathcal{B}}^{0} \backslash\{T\}\right) \cup \mathcal{Q}_{\mathcal{C}}^{0} & \text { if } T \in \mathcal{Q}_{\mathcal{B}}^{0} \\ \mathcal{Q}_{\mathcal{B}}^{0} & \text { otherwise }\end{cases}$

$\Delta_{\mathcal{B}^{\prime}}=\left(\Delta_{\mathcal{B}} \backslash\{\langle R, F, T\rangle\}\right) \cup\left\{\left\langle R, F, T^{\prime}\right\rangle \mid T^{\prime} \in \mathcal{Q}_{\mathcal{C}}^{0}\right\} \cup \Delta_{\mathcal{C}}$

is a

\section{Emptiness Check of $\wp$-UTGBA}

A generalized Büchi automaton accepts a run (i.e., is nonempty) if it contains a reachable cycle in which all acceptance conditions appear. The emptiness check algorithms that have been devised to check this condition can be distinguished into two classes [5]:

- Nested Depth-First Searches (NDFS) algorithms will use a first DFS to find a state from which several DFS will be nested to find cycles around this state that visit each acceptance condition [12].

- Strongly-Connected-Component-based (SCC) algorithms will decompose the automaton into SCC and look for a reachable SCC in which all acceptance conditions appear.

NDFS algorithms have long been favored to SCC-based algorithms because of their better memory footprint (a couple of extra bits per state versus an integer). However it has 


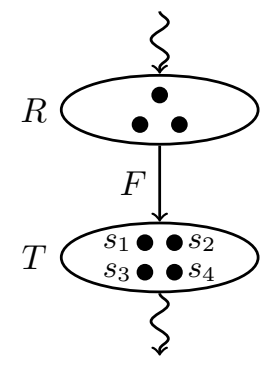

$(\mathcal{B})$

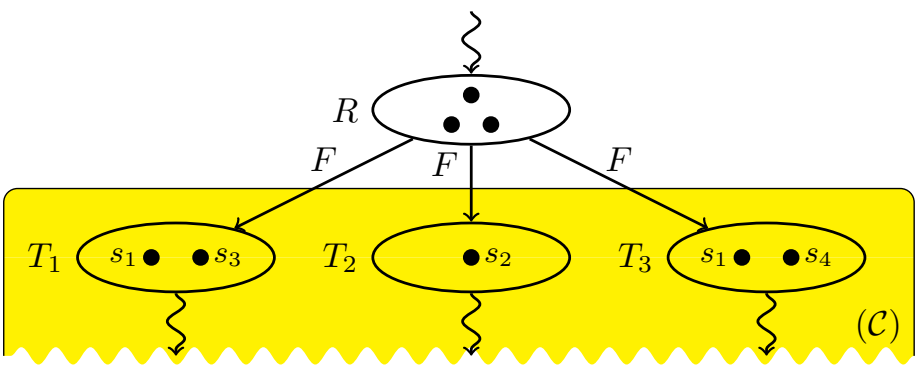

$\left(\mathcal{B}^{\prime}\right)$

Figure 2. Example of decomposition of a transition $\langle R, F, T\rangle$ using proposition 3.

been pointed out that these extra data are negligible compared to what it takes to represent a state of a system (often hundreds of bytes) [11]. Furthermore, SCC-based algorithms have several advantages over NDFS: they need to explore less states to detect non-empty automata [7, 5], and they will deal with generalized acceptance conditions for free [5]. The algorithm we present here is derived from that of Couvreur [3], which is SCC-based.

\subsection{Original algorithm}

The idea is to enumerate all the maximal strongly connected components (MSCC) of an automaton. Any graph contains at least one MSCC without an outgoing arc, so to list all MSCCs, we should find such a terminal MSCC, remove it from the graph, and list all MSCCs of the resulting graph. To do so, the algorithm performs a depth-first search (DFS) of the automaton. While doing so, it maintains a stack of SCCs traversed by the DFS stack. As new transitions are visited, the SCC stack may be augmented or compacted. When an SCC is popped off the stack, meaning it is terminal, we check whether it is accepting: if that is the case, the algorithm terminates, otherwise all the states of this component are marked as "removed" so that whenever the DFS hits one of them again it can ignore it.

\subsection{Adaptation to $\wp$-UTGBA}

The new algorithm differs from the original in two points. First, the check for removed states is generalized: any removed state $D$ can indeed be ignored by the DFS, but so can any state $T \subseteq D$ ! This is thanks to proposition 2 .

Fig. 3 illustrates the second difference. Consider automaton $\mathcal{B}_{1}$ where the DFS is examining the transition $\langle R, F, T\rangle$ going to a new state $T$. Notice that there exists a state $D$ in the search stack (or more generally in any SCC on the search stack) such that $D \subseteq T$. From the point of view of the underlying automaton it means some states in $R$ can reach those in $D$ and vice-versa, so they all belong to

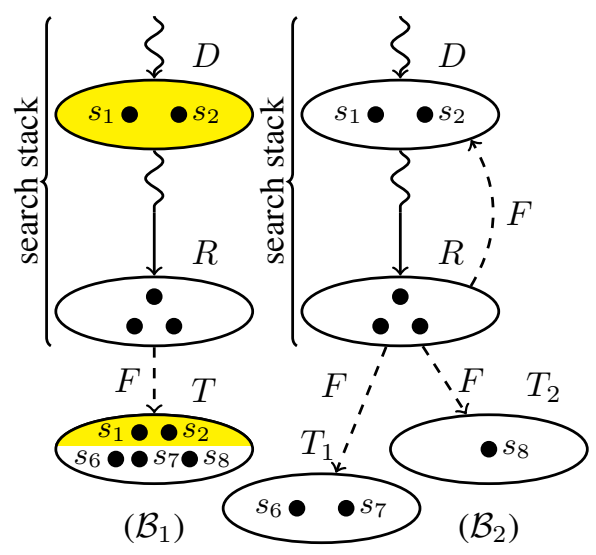

Figure 3. Inclusion checks in the search stack. We rewrite $\mathcal{B}_{1}$ to $\mathcal{B}_{2}$.

the same SCC. For the emptiness check it would be beneficial to split the transition $\langle R, F, T\rangle$ as in $\mathcal{B}_{2}$ : it explicits the loop on the SCC and reuses previously seen states. Such a decomposition is correct thanks to proposition 3 , but it has additional constraints we now formalize.

Let $\mathcal{B}=\left\langle\mathcal{Q}_{\mathcal{B}}, \mathcal{Q}_{\mathcal{B}}^{0}, \mathcal{F}, \Delta_{\mathcal{B}}\right\rangle$ be a $\wp$-UTGBA over some $\mathcal{A}$. $\operatorname{Decomp}(\mathcal{B},\langle S, F, T\rangle, D)$ is an operation that should perform a decomposition like in proposition 3. Besides $\mathcal{B}$ and $\langle S, F, T\rangle$, which have the same purpose as in the definition, the argument $D$ is a state of $\mathcal{B}$ that is also a subset of $T$. Decomp should build the required automaton $\mathcal{C}$ with two additional constraints:

- we want $D \in \mathcal{Q}_{\mathcal{C}}^{0}$ (and the other states of $\mathcal{Q}_{\mathcal{C}}^{0}$ will by definition complete $T$ ),

- and $\Delta_{\mathcal{C}}$ should not add transitions to the states of $\mathcal{B}$, i.e., $\left\{\left\langle S, F, S^{\prime}\right\rangle \in \Delta_{\mathcal{C}} \mid S \in \operatorname{Reach}(\mathcal{B})\right\} \subseteq \Delta_{\mathcal{B}}$.

Decomp returns a pair $\mathcal{B}^{\prime}, \mathcal{Q}_{\mathcal{C}}^{0}$ : the new automaton, and the initial states of $\mathcal{C}$. (Since in practice we build $\mathcal{B}$ on the fly, as needed by the emptiness check, what really matters is that Decomp doesn't add any transition to the part of $\mathcal{B}$ 
already seen by the emptiness check, so it can continue from the result of Decomp as if it had started from it.)

The complete algorithm presented in Fig. 4 requires three operations on the structure of the states: tests for equality and inclusion, and the decomposition. We denote them respectively, $=, \subseteq$ and Decomp. These operations are the only steps of the algorithm that have to be tailored to the encoding of the states.

\subsection{Correctness of this algorithm}

We only sketch enough of the actual proof [1] to be able to understand the algorithm. At any point of the execution we denote the contents of the todo and $S C C$ stacks by:

$$
\begin{aligned}
\text { todo } & =\left\langle\text { state }_{0}, \text { succ }_{0}\right\rangle\left\langle\text { state }_{1}, \text { succ }_{1}\right\rangle \cdots\left\langle\text { state }_{m}, \text { succ }_{m}\right\rangle \\
S C C & =\left\langle\text { root }_{0}, \text { la }_{0}, \text { acc }_{0}, \text { rem }_{0}\right\rangle \cdots\left\langle\text { root }_{n}, \text { la }_{n}, \text { acc }_{n}, \text { rem }_{n}\right\rangle
\end{aligned}
$$

todo is a DFS stack of pairs $\langle$ state, succ $\rangle$ where succ is the set of outgoing transitions of state that haven't been considered yet. We call state . $_{\text {. }}$ states $s_{m}$ the search path.

Each tuple in $S C C$ represents a strongly connected component traversed by the search path. root $_{i}$ is the number of the first state of the component visited by the algorithm, and together with $H$ (a map that numbers each visited state) it allows to define the set $\mathfrak{S}_{i}$ of states belonging to the $i^{\text {th }} \mathrm{SCC}$ as follows:

$\mathfrak{S}_{i}=\left\{s \in \mathcal{Q}_{\mathcal{B}} \mid \operatorname{root}_{i} \leqslant H[s]<\operatorname{root}_{i+1}\right\}$ for $0 \leqslant i<n$ $\mathfrak{S}_{n}=\left\{s \in \mathcal{Q}_{\mathcal{B}} \mid \operatorname{root}_{n} \leqslant H[s]\right\}$

$a c c_{i}$ is the set of acceptance conditions traversed by transitions between states of $\mathfrak{S}_{i} . l a_{i}$ are the acceptance conditions on the transition between the $(i-1)^{\text {th }}$ and the $i^{\text {th }}$ components. The resulting chain of SCC is depicted by Fig. 5. Finally $\mathrm{rem}_{i}$ is a set of states to be removed when the component is popped, as we will see later.

The states of the automaton $\mathcal{B}$ being checked are partitioned into three sets:

- The active states are those which are keys of $H$ and have a nonzero value,

- the removed states are those which are keys of $H$ and have a value of 0 ,

- the unexplored states are those that are not keys of $H$.

Initially, all states are unexplored. The function "DFSpush" is the only place a state can switch from unexplored to active, and the function "DFSpop" is the only place where it can switch from active to removed.

The following invariants are preserved at every line of 50 "main" [1]:

Proposition 4. $m \geqslant n$ (in the above notation for todo and $S C C$ ) and there exists a strictly increasing function $f$ such that $\forall i \leqslant n$ root $_{i}=H\left[\right.$ state $\left._{f(i)}\right]$. In

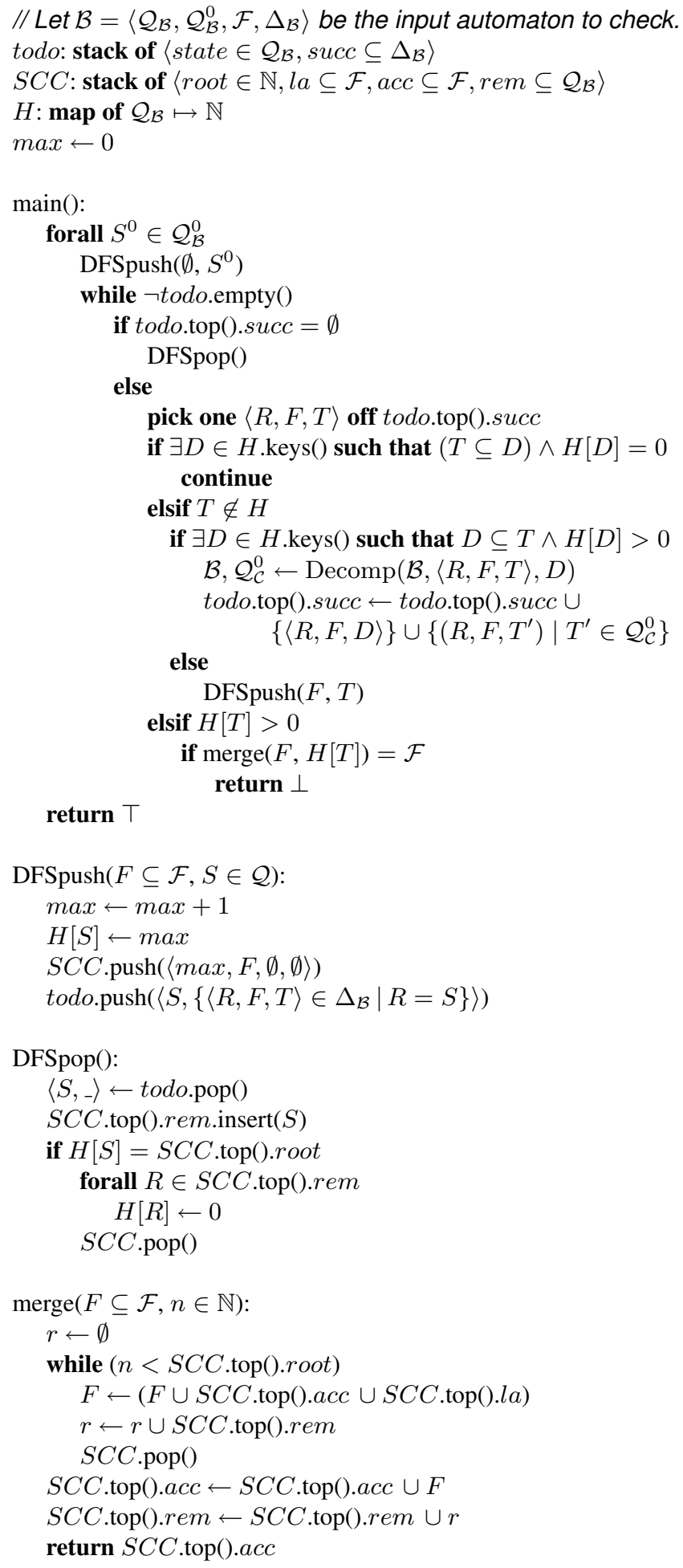

Figure 4. Emptiness check of a $\wp$-UTGBA. 


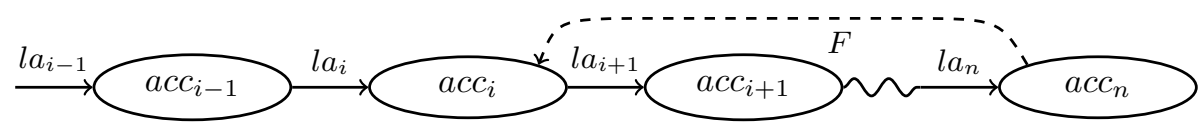

Figure 5. The meaning of $l a$ and $a c c$ in $S C C$.

other words, root $_{0}$, root $_{1}, \ldots$, root $_{n}$ is a subsequence of $H\left[\right.$ state $\left._{0}\right], H\left[\right.$ state $\left._{1}\right], \ldots, H\left[\right.$ state $\left._{m}\right]$. (I.e., the roots of the strongly connected components are on the search path of the depth-first search, and in the same order.)

Proposition 5. For any $i \leqslant n$ the subgraph induced by the states of $\mathfrak{S}_{i}$ is a SCC. Furthermore there exists a cycle in this SCC that visits all the acceptance conditions of acc . $_{\text {. }}$ Finally $\mathfrak{S}_{0}, \mathfrak{S}_{1}, \cdots, \mathfrak{S}_{n}$ is a partition of the set of active states.

Proposition 6. $\forall i<n, \exists s \in \mathfrak{S}_{i},\left\langle s, l a_{i+1}\right.$, state $\left._{f(i+1)}\right\rangle \in$ $\Delta_{\mathcal{B}}$.

Proposition 7. For any $i<n$, rem $_{i}$ holds all states of $\mathfrak{S}_{i}$ not on the search path.

Proposition 8. For any removed state $q, \operatorname{Acc}(\mathcal{B}[\{q\}])=\emptyset$.

The first two propositions guarantee that if the algorithm finds an $i$ such that $a c c_{i}=\mathcal{F}$, it corresponds to a reachable (prop. 4) accepting component (prop. 5). The last proposition justifies that no accepting run exists if the algorithm has removed all the states.

\subsection{Counterexamples}

When verifying a model by the automata theoretic approach, the presence of an accepting run means that there exists an execution of the modeled system that invalidates the property being checked, i.e., a counterexample. Therefore whenever the emptiness check exits with $\perp$, meaning the automaton has an accepting run, the user usually wants to see such a run to debug the model (or the property).

To produce a genuine accepting run from the stack of SCCs, these SCCs have to be searched again. The method described by Couvreur et al. [5] could be used only with the guarantee it would visit the same states that the emptiness check visited.

In our case, doing so seems hard because the computation of the successors of a state using inclusion and decomposition depend on the value of $H$.keys() which has evolved. Besides, it would only give a counterexample using states of $\mathcal{B}$ while the user will prefer a counterexample using states of $\mathcal{A}$.

Our suggestion is that once the emptiness check of $\mathcal{B}$ has failed, we search a counterexample in $\mathcal{A}$, but using the data structures computed by the emptiness check of $\mathcal{B}$ to narrow the search. Computing the set of states of each SCC is easily done by unwinding the todo and $S C C$ search stacks: the set of states that belong to the top-most SCC is $\mathfrak{S}_{n}=\operatorname{rem}_{n} \cup$ $\left\{\right.$ state $_{f\left(\text { root }_{n}\right)}$, state $_{f\left(\text { root }_{n}\right)+1}, \ldots$, state $\left._{m}\right\}$. The set of states from the previous SCC are then $\mathfrak{S}_{n-1}=\operatorname{rem}_{n-1} \cup$ $\left\{\right.$ state $_{f\left(\text { root }_{n-1}\right)}$, state $_{f\left(\text { root }_{n-1}\right)+1}, \ldots$, state $\left._{f\left(\text { root }_{n}\right)-1}\right\}$, etc. Checking whether a state $s \in \mathcal{Q}_{\mathcal{A}}$ belongs to the $i^{\text {th }}$ SCC then amounts to testing whether $\exists \mathfrak{S} \in \mathfrak{S}_{i}$ such that $s \in \mathfrak{S}$. It is thus possible to constraint the search in $\mathcal{A}$ to remain inside these SCCs.

\section{Approximative Emptiness Check}

Consider Fig. 3 again. In the previous section we have seen that at lines 18-21 the algorithm of Fig. 4 takes the situation depicted by automaton $\mathcal{B}_{1}$, where the emptiness check reaches a state $T \supseteq D$ such that $D$ belongs to the search stack, and translates that into $\mathcal{B}_{2}$ to reuse existing states and build SCCs as soon as possible. We proved that this transformation preserves the result of the emptiness check $\left(\mathcal{B}_{1} \emptyset\right.$-equivalent to $\left.\mathcal{B}_{2}\right)$.

We now turn to the situation $\mathcal{B}_{3}$ on Fig. 6 , where the emptiness check examines a transition $\langle R, F, T\rangle$ such that $T \subseteq D$ and $D$ is in the search stack. We can rewrite this transition as $\langle R, F, D\rangle$, as depicted by $\mathcal{B}_{4}$, by replacing lines $18-20$ of Fig. 4 by:

$$
\begin{aligned}
& \text { if } \exists D \in H . \text { keys() such that } T \subseteq D \wedge H[D]>0 \\
& \quad / / \text { Note the order of } T \text { and } D \text { above. } \\
& \text { todo.top().succ } \leftarrow \text { todo.top().succ } \cup\{\langle R, F, D\rangle\}
\end{aligned}
$$

Assume that $\mathcal{B}_{3}$ is a $\wp$-UTGBA over some $\mathcal{A}$. Note that the above transformation breaks property (5) of definition 5, because $s_{3}$ and $s_{4}$ have no predecessor in $R$; so $\mathcal{B}_{4}$ is not a $\wp$-UTGBA over $\mathcal{A}$. However by adding some transitions to $\mathcal{A}$ to please property (5) it is possible to derive an $\mathcal{A}^{\prime}$ such that $\mathcal{B}_{4}$ is a $\wp$-UTGBA over $\mathcal{A}^{\prime}$.

Therefore if the emptiness check algorithm finds an accepting component in $\mathcal{B}_{4}$, there is an accepting run in $\mathcal{A}^{\prime}$ but not necessarily in $\mathcal{A}$. However since runs of $\mathcal{A}$ are also runs of $\mathcal{A}^{\prime}$, if the algorithm does not find any accepting component in $\mathcal{B}_{4}$, no accepting run exists in $\mathcal{A}$ and $\mathcal{A}^{\prime}$.

In other words, this modified algorithm returns "empty" or "I don't know". As we will show in Section 5.4 this transformation is a lot faster than the other ("correct") one presented in Section 3. Since model-checking is mainly interested in ensuring that some automaton is empty, it makes sense to try this semi-decision procedure first and fall back to the "correct" procedure if the answer isn't "empty". 


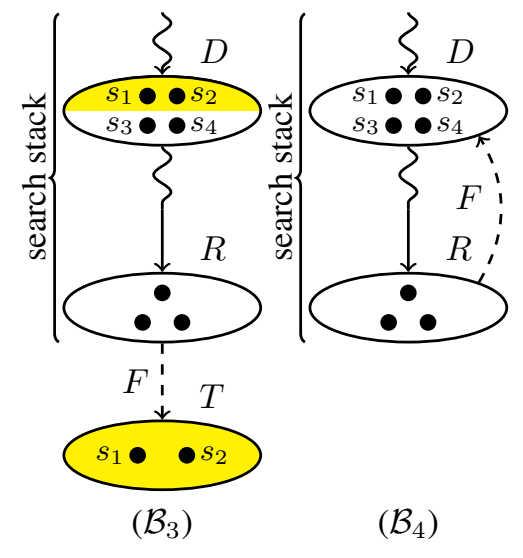

Figure 6. Inclusion checks in the search stack. We rewrite $\mathcal{B}_{3}$ to $\mathcal{B}_{4}$.

Note by the way that if the modified algorithms return $\perp$ but no inclusion has been done in the stack (one could count the number of times line 20 has been executed), then the automaton actually is nonempty.

\section{Application to Symmetries}

In this section we show how to exploit symmetries to construct a UTGBA $\mathcal{B}$ that is a $\wp$-UTGBA over some automaton $\mathcal{A}$. We first define $\mathcal{A}$, the synchronized product of a transition system $\mathcal{T}$, representing the behavior of a system, and a Transition-based Generalized Büchi Automaton $\mathcal{P}$, representing the property to check.

Definition 7 (Labelled transition system). A labelled transition system is a tuple $\mathcal{T}=\left\langle\mathcal{Q}_{\mathcal{T}}, \mathcal{Q}_{\mathcal{T}}^{0}, \Sigma, \Delta_{\mathcal{T}}\right\rangle$, where

- $\mathcal{Q}_{\mathcal{T}}$ is a finite set of states,

- $\mathcal{Q}_{\mathcal{T}}^{0} \subseteq \mathcal{Q}_{\mathcal{T}}$ is the set of initial states,

- $\Sigma=2^{A P}$ is an alphabet, where AP is the set of atomic propositions,

- $\Delta_{\mathcal{T}} \subseteq \mathcal{Q}_{\mathcal{T}} \times \Sigma \times \mathcal{Q}_{\mathcal{T}}$ is a transition relation such that $\forall\left\langle s_{1}, p_{1}, d_{1}\right\rangle,\left\langle s_{2}, p_{2}, d_{2}\right\rangle \in \Delta_{\mathcal{T}}, p_{1}=p_{2} \Longleftrightarrow$ $\left(s_{1}, d_{1}\right)=\left(s_{2}, d_{2}\right)$.

The set of reachable states $\operatorname{Reach}(\mathcal{T})$ is defined as usual. The latter condition on $\Delta_{\mathcal{T}}$ means that each transition is uniquely defined by its label (it is always possible to add more atomic propositions to the system to satisfy this constraint).

Definition 8 (Transition-based Generalized Büchi Automaton). A TGBA is a Büchi automaton with labels and generalized acceptance conditions on transitions. It is defined as a tuple $\mathcal{P}=\left\langle\mathcal{Q}_{\mathcal{P}}, \mathcal{Q}_{\mathcal{P}}^{0}, \Sigma, \mathcal{F}, \Delta_{\mathcal{P}}\right\rangle$, where
- $\mathcal{Q}_{\mathcal{P}}$ is a finite set of states,

- $\mathcal{Q}_{\mathcal{P}}^{0} \subseteq \mathcal{Q}_{\mathcal{P}}$ is a set of initial states,

- $\Sigma=2^{A P}$ is an alphabet,

- $\mathcal{F}$ is a finite set of acceptance conditions,

- $\Delta_{\mathcal{P}} \subseteq \mathcal{Q}_{\mathcal{P}} \times \Sigma \times 2^{\mathcal{F}} \times \mathcal{Q}_{\mathcal{P}}$ is the transition relation, where each transition is labelled by a letter of $\Sigma$ and a set of acceptance conditions of $\mathcal{F}$.

Definition 9 (Synchronized product). The synchronized product between $\mathcal{T}=\left\langle\mathcal{Q}_{\mathcal{T}}, \mathcal{Q}_{\mathcal{T}}^{0}, \Sigma, \Delta_{\mathcal{T}}\right\rangle$ and $\mathcal{P}=$ $\left\langle\mathcal{Q}_{\mathcal{P}}, \mathcal{Q}_{\mathcal{P}}^{0}, \Sigma, \mathcal{F}, \Delta_{\mathcal{P}}\right\rangle$ is the UTGBA $\mathcal{A}=\mathcal{T} \otimes \mathcal{P}$ defined by $\mathcal{A}=\left\langle\mathcal{Q}_{\mathcal{A}}, \mathcal{Q}_{\mathcal{A}}^{0}, \mathcal{F}, \Delta_{\mathcal{A}}\right\rangle$, where

- $\mathcal{Q}_{\mathcal{A}}=\mathcal{Q}_{\mathcal{T}} \times \mathcal{Q}_{\mathcal{P}}$ is the set of states,

- $\mathcal{Q}_{\mathcal{A}}^{0}=\mathcal{Q}_{\mathcal{T}}^{0} \times \mathcal{Q}_{\mathcal{P}}^{0}$ is the set of initial states,

- $\Delta_{\mathcal{A}} \subseteq \mathcal{Q}_{\mathcal{A}} \times 2^{\mathcal{F}} \times \mathcal{Q}_{\mathcal{A}}$ is the transition relation between states such that

$\exists\left\langle\langle s, q\rangle, F,\left\langle s^{\prime}, q^{\prime}\right\rangle\right\rangle \in \Delta_{\mathcal{A}} \quad$ iff $\exists\left\langle s, p, s^{\prime}\right\rangle \quad \in$ $\Delta_{\mathcal{T}}, \exists\left\langle q, p^{\prime}, F, q^{\prime}\right\rangle \in \Delta_{\mathcal{P}}$ and $p=p^{\prime}$.

\subsection{Symmetry-based Construction of a $\wp-$ UTGBA}

Now we construct $\mathcal{B}$, a $\wp$-UTGBA over $\mathcal{A}=\mathcal{T} \otimes \mathcal{P}$, using a technique introduced by Haddad et al. [8] but adapted to transition-based automata. The idea is to exploit the symmetries of $\mathcal{T}$ in addition to those of the $\operatorname{arcs}$ of $\mathcal{P}$, to gather sets of nodes of $\mathcal{A}$.

Since these symmetries sit on group theory, we recall some elementary notions.

Definition 10. Let $(G, \circ)$ be a group with a neutral element $i d$, and let $E$ be a set.

- An action of $G$ over $E$ is a mapping $G \times E \mapsto E$ such that the image $(g, e)$ denoted by g.e fulfills $\forall e \in E$ : $i d . e=e$ and $\forall g, g^{\prime} \in G,\left(g \circ g^{\prime}\right) . e=g \cdot\left(g^{\prime} . e\right)$.

- The isotropy subgroup $G_{E^{\prime}}$ of a subset $E^{\prime} \subseteq E$ is defined by $G_{E^{\prime}}=\left\{g \in G \mid \forall e \in E^{\prime}\right.$, g.e $\left.\in E^{\prime}\right\}$.

- For a subgroup $H$ of $G$ (denoted $H<G$ ), the orbit $H$.e of $e \in E$ under $H$ is H.e $=\{g . e \mid g \in H\}$.

- An action $g$ of $G$ can be straightforwardly extended to the powerset of $E$. For any $E^{\prime} \subseteq E, g . E^{\prime}=\{g . e \mid$ $\left.e \in E^{\prime}\right\}$.

We can now characterize a symmetric transition system with respect to a group.

Definition 11 (Symmetric transition system with respect to a group). Let $\mathcal{T}=\left\langle\mathcal{Q}_{\mathcal{T}}, \mathcal{Q}_{\mathcal{T}}^{0}, \Sigma, \Delta_{\mathcal{T}}\right\rangle$ be a transition system and $G$ be a group acting on $A P . \mathcal{T}$ is said to be symmetric with respect to $G$ iff every transition of $\mathcal{T}$ has a "symmetric" transition with respect to any element of $G$ and the action of $G$ is congruent with respect to the transition relation: $\forall g \in G, \forall\left\langle s_{1}, p_{1}, d_{1}\right\rangle \in \Delta_{\mathcal{T}}, \exists\left\langle s_{2}, p_{2}, d_{2}\right\rangle \in \Delta_{\mathcal{T}}$ such that 


$$
\left\{\begin{array}{l}
s_{1} \in \mathcal{Q}_{\mathcal{T}}^{0} \Longleftrightarrow s_{2} \in \mathcal{Q}_{\mathcal{T}}^{0}, \\
p_{2}=g \cdot p_{1} \text { and } \\
\forall\left\langle s_{1}^{\prime}, p_{1}^{\prime}, d_{1}^{\prime}\right\rangle \in \Delta_{\mathcal{T}}, s_{1}^{\prime}=d_{1}, \exists\left\langle s_{2}^{\prime}, p_{2}^{\prime}, d_{2}^{\prime}\right\rangle \in \Delta_{\mathcal{T}} \text {, s.t. } \\
\quad s_{2}^{\prime}=d_{2} \text { and } p_{2}^{\prime}=\text { g.p } p_{2}
\end{array}\right.
$$

The action of the group on $A P$ is extended to $\operatorname{Reach}(\mathcal{T})$ by denoting g.s the unique $s_{2}$ such that $\forall g \in G, \forall\left\langle s_{1}, p_{1}, d_{1}\right\rangle \in \Delta_{\mathcal{T}}, s_{1}=s, \exists d_{2} \in$ $\mathcal{Q}_{\mathcal{T}},\left\langle s_{2}, g . p_{1}, d_{2}\right\rangle \in \Delta_{\mathcal{T}}$. (The uniqueness is due to the constraint on $\Delta_{\mathcal{T}}$ in definition 7.)

Because $G$ is group, a consequence of this definition is that $G \cdot \mathcal{Q}_{\mathcal{T}}^{0}=\mathcal{Q}_{\mathcal{T}}^{0}$.

These definitions allow us to give a possible construction of a $\mathcal{B}$.

Definition 12 (Symbolic Synchronized Product). Let $\mathcal{T}=$ $\left\langle\mathcal{Q}_{\mathcal{T}}, \mathcal{Q}_{\mathcal{T}}^{0}, \Sigma, \Delta_{\mathcal{T}}\right\rangle$ be a transition system symmetric w.r.t. a group $G, \mathcal{P}=\left\langle\mathcal{Q}_{\mathcal{P}}, \mathcal{Q}_{\mathcal{P}}^{0}, \Sigma, \mathcal{F}, \Delta_{\mathcal{P}}\right\rangle$. The Symbolic Synchronized Product of $\mathcal{T}$ and $\mathcal{P}$ is a UTGBA $\mathcal{B}=$ $\left\langle\mathcal{Q}_{\mathcal{B}}, \mathcal{Q}_{\mathcal{B}}^{0}, \mathcal{F}, \Delta_{\mathcal{B}}\right\rangle$ where:

- $\mathcal{Q}_{\mathcal{B}}^{0}=\left\{\langle G, G . s, q\rangle \mid s \in \mathcal{Q}_{\mathcal{T}}^{0}, q \in \mathcal{Q}_{\mathcal{P}}^{0}\right\}$

- $\mathcal{Q}_{\mathcal{B}}=\mathcal{Q}_{\mathcal{B}}^{0} \cup \mathcal{V}$ where $\mathcal{V}$ is the set of tuples of the form $\langle H, O, q\rangle$ such that $H<G, O \subseteq \operatorname{Reach}(\mathcal{T}), q \in \mathcal{Q}_{\mathcal{P}}$, and $H . O=O$.

- $\Delta_{\mathcal{B}}$ is defined by construction as follows: $\left\langle\langle H, O, q\rangle, F,\left\langle H^{\prime}, O^{\prime}, q^{\prime}\right\rangle\right\rangle \in \Delta_{\mathcal{B}}$ iff

$\exists\left(s, s^{\prime}, p, p^{\prime}, F\right) \in O \times O^{\prime} \times \Sigma \times \Sigma \times 2^{\mathcal{F}}$ such that $\left\langle s, p, s^{\prime}\right\rangle \in \Delta_{\mathcal{T}},\left\langle q, p^{\prime}, F, q^{\prime}\right\rangle \in \Delta_{\mathcal{P}}$, and $p=p^{\prime}$. Then $O^{\prime}=\left(H \cap G_{p^{\prime}}\right) \cdot s^{\prime}$ and $H^{\prime} \subseteq G_{O^{\prime}}$.

If $\mathcal{A}=\mathcal{T} \otimes \mathcal{P}=\left\langle\mathcal{Q}_{\mathcal{A}}, \mathcal{Q}_{\mathcal{A}}^{0}, \mathcal{F}, \Delta_{\mathcal{A}}\right\rangle$, any state $\langle H, O, q\rangle \in \mathcal{Q}_{\mathcal{B}}$ of $\mathcal{B}$ represents the set $\left\{\left\langle x, q^{\prime}\right\rangle \in \mathcal{Q}_{\mathcal{A}} \mid\right.$ $\left.x \in O \wedge q^{\prime}=q\right\}$ of states of $\mathcal{A}$. Hence we can write $\left\langle x, q^{\prime}\right\rangle \in\langle H, O, q\rangle$, and this allows us to prove that $\mathcal{B}$ is a $\wp$-UTGBA over $\mathcal{A}[1]$.

\subsection{Asymmetric Transition Systems}

The method, as described here, is heavily dependent on the global symmetries of the transition system $\mathcal{T}$ (i.e., on the group $G$ ). The bigger $G$ is, the better the achieved reduction is. On a transition system mostly asymmetric, $G$ will be very small, maybe the identity (i.e., no symmetries at all), and consequently the subgroups $H<G$ computed for each node will allow even less reductions.

There is one way to handle an asymmetric transitions system $\mathcal{T}$ with this method: it is to rewrite it as a composition $\mathcal{T}_{S} \otimes \mathcal{C}$ where $\mathcal{T}_{S}$ is globally symmetric w.r.t. a large $G$ and $\mathcal{C}$ is a constraint automaton such that $\mathcal{T}_{S} \otimes \mathcal{C}=\mathcal{T}$. Now, instead of constructing the symbolic synchronized product method of $\mathcal{T} \otimes \mathcal{P}$, we can construct it for $\mathcal{T}_{S} \otimes \mathcal{P}_{C}$ where
$\mathcal{P}_{C}=\mathcal{C} \otimes \mathcal{P}$. In other words, we shifted all asymmetries from the system automaton to the property automaton. This works because the method does not require a symmetric property automaton.

Haddad et al. [8] show one way to construct $\mathcal{T}_{S}$ and $\mathcal{C}$ from $\mathcal{T}$, while we are using a more optimal transition-based construction which is yet unpublished.

\subsection{Operations for the emptiness check}

For this construction to make sense (memory-wise), the set $O$ of a state $\langle H, O, q\rangle$ must never be stored explicitly. In our implementation this is achieved by using a modified version of the symbolic representation of Well Formed Petri-Nets [2]. This data structure supports the following operations.

Let $T=\left\langle H_{1}, O_{1}, q_{1}\right\rangle$ and $D=\left\langle H_{2}, O_{2}, q_{1}\right\rangle$ be two states of $\mathcal{B}$. Since the sets $O_{i}$ are not stored explicitly we cannot compare states with different $H_{i}$ unless they are expanded into the set of states of $\mathcal{A}$ they represent. To avoid this explicit expansion we introduce the following operation that we can use to unify the $H_{i}$.

The refinement of $\left\langle H_{1}, O_{1}, q_{1}\right\rangle$ w.r.t. $H_{2}$ is the finite set $\operatorname{Ref}\left(\left\langle H_{1}, O_{1}, q_{1}\right\rangle, H_{2}\right)=\left\{\left\langle H_{1} \cap H_{2}, O_{i}, q_{1}\right\rangle \mid i \in \mathbb{N}\right\}$ such that $\forall i,\left(H_{1} \cap H_{2}\right) . O_{i}=O_{i}$, and $\bigcup_{i} O_{i}=O_{1}$.

This allows us to check the inclusion of two states with: $\left\langle H_{1}, O_{1}, q_{1}\right\rangle \subseteq\left\langle H_{2}, O_{2}, q_{1}\right\rangle$ iff $\operatorname{Ref}\left(\left\langle H_{1}, O_{1}, q_{1}\right\rangle, H_{2}\right) \subseteq$ $\operatorname{Ref}\left(\left\langle H_{2}, O_{2}, q_{1}\right\rangle, H_{1}\right)$.

Seeking visited states that include others (as on lines 15 and 18 of Fig. 4) can be sped up using a two level hashtable. Let $G$ be the group acting on $A P$ such that $\forall p \in$ $A P, G . p=A P$. For a state $\left\langle H_{1}, O_{1}, q_{1}\right\rangle$, pick an $s \in O_{1}$ : $G . s$ is the coarsest equivalence class in which $s$ can belong. We use G.s as a key for our first-level hash table and $q_{1}$ as a key for the second level. Therefore when looking for states that include $\left\langle H_{1}, O_{1}, q_{1}\right\rangle$, we only need to look through the states that share the same G.s and $q_{1}$.

$\operatorname{Decomp}(\mathcal{B},\langle R, F, T\rangle, D)$ is achieved using the refinement above. $T$ is refined with respect to $D$ 's $H_{2}$, and $D$ is refined with respect to $H_{1}$, so we can compute the difference: $\left\{T_{i}\right\}_{i}=\operatorname{Ref}\left(\left\langle H_{1}, O_{1}, q_{1}\right\rangle, H_{2}\right) \backslash$ $\operatorname{Ref}\left(\left\langle H_{2}, O_{2}, q_{1}\right\rangle, H_{1}\right)$. (The algorithm is improved by grouping some of these $T_{i} \mathrm{~s}$.)

\subsection{Performance}

The symbolic synchronized product of definition 12 has been implemented using the core of GreatSPN ${ }^{1}$ [2], and the emptiness checks we presented are implemented in $\operatorname{Spot}^{2}$ [6]. Connecting the two tools allowed us to compare different techniques.

\footnotetext{
${ }^{1}$ http://www.di.unito.it/ $\sim$ greatspn/

${ }^{2}$ http://spot.1ip6.fr/
} 


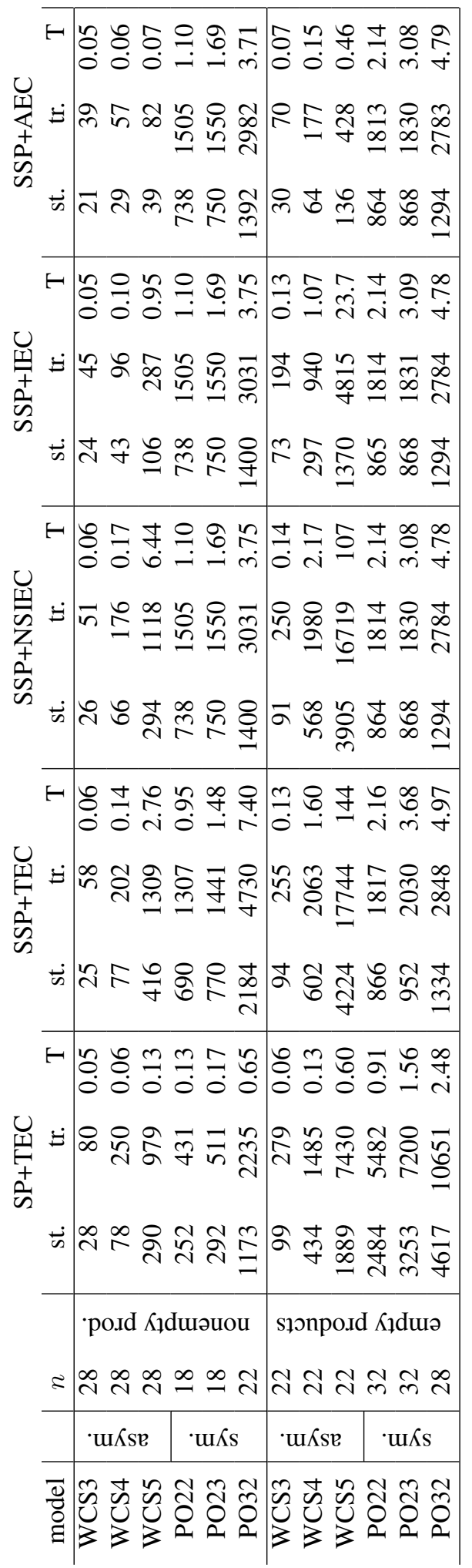

Table 1. States (st.) and transitions (tr.) explored by each algorithm on different models, and seconds (T) taken. All averaged on $n$ properties.
Table 1 presents some measurements on two parametrized models, WCS [2] and PO [10], that were chosen for their symmetric or asymmetric properties: PO is a completely symmetric model (objects of similar nature behave identically), while WCS is an asymetric model (objects behave differently). In both cases increasing the parameter increases the number of states of the system. Each of these models was synchronized against 50 randomly generated property automata: the table has been split to average the cases where the resulting product is empty separately from the cases where it is not. The reason is that the emptiness check has to check all states of an empty product, but can abort early if it is nonempty. The column $n$ shows how many of the 50 cases were empty or not.

The abbreviations in the headers refer to how the product was constructed and checked for emptiness. SP is the synchronized product of definition 9 while SSP designates the symbolic synchronized product of definition 12. SP's states are not sets, so it is checked with a traditional emptiness check (TEC) similar to the one of Fig. 4 but without any inclusion check or decomposition. IEC designates the emptiness check of Fig. 4. NSIEC is the same algorithm without lines 18-21 (i.e., No Stack Inclusion). Finally, AEC designates the approximative emptiness check of Section 4.

We observe that although SP is a lot faster than SSP, it visits many more states and hence requires a lot more memory. The different versions of our emptiness check algorithm can be compared in the four SSP columns: on the WCS model, adding inclusion checks in the removed states (NSIEC) reduces the size of the explored automaton (compared to TEC), and adding inclusion checks in the search stack (IEC) reduces the automaton further. Therefore, although the decomposition operation is costly (time-wise) it really helps reduce the memory footprint of the modelchecking. The last column shows that approximation is indeed faster and constructs less states than all other methods (and yielded no false negatives in these experiments). On the PO model, the new emptiness check algorithms are not significantly better because the symmetric nature of the model offers little occasion for inclusion; still it can be seen that they do not incur any overhead.

Finally Fig. 7 shows the memory consumption of our implementation of the latest emptiness-check algorithm w.r.t. the traditional approach.

\section{Conclusion}

In this paper, we presented two novel emptiness check algorithms dealing with automata whose states are sets, and exploiting inclusions between these sets.

The results we obtained on the symmetry-based construction indicate that using inclusion and decomposition reduces the number of states by a great factor to the detri- 


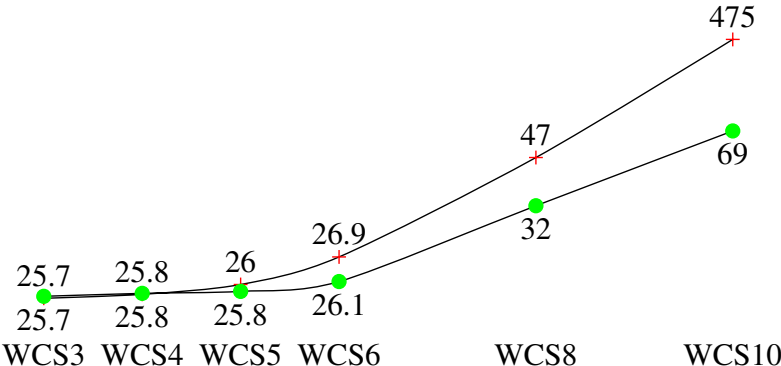

Figure 7. Average Memory used (in MB, on a log scale) by our implementations of SP+TEC (top) and SSP+AEC (bottom) to check empty products of increasing WCS models.

ment of the time. Actually, the loss of time is due to the way the states are encoded. Because this technique calls for a representation of sets that allow fast inclusion checks and decomposition, we expect that encoding sets with BDDs or DDDs [13] would improve the situation.

However this application should not occlude the fact that our algorithms have been developed in a more generic framework: any technique that can construct an automaton satisfying the five constraints of definition 5 could be used with these emptiness checks.

We believe it might be even possible to relax these constraints in order to support more methods, like the previously mentioned unfolding graphs [4] or observation graphs [9], that construct some "set automata" that do not strictly satisfy our conditions.

Another area that could be explored is the use of inclusion checks in NDFS emptiness checks. This doesn't seem easy because NDFS algorithms will visit a state several times and require that the set of successors (re-)computed for a state remain the same: this is not guaranteed if the inclusion checks use a $H$.keys() that changes. This is the same problem faced with counterexample generation in section 3.4, so ideas for either area could benefit the other.

\section{References}

[1] S. Baarir and A. Duret-Lutz. Emptiness check of powerset Büchi automata. Technical report 2006/003, Université Pierre et Marie Curie, LIP6-CNRS, Paris, France, Oct. 2006.

[2] S. Baarir, S. Haddad, and J.-M. Ilié. Exploiting partial symmetries in well-formed nets for the reachability and the linear time model checking problems. In Proc. of WODES'04, pages 223-228, Sept. 2004.

[3] J.-M. Couvreur. On-the-fly verification of temporal logic. In Proc. FM'99, volume 1708 of $L N C S$, pages 253-271. Springer-Verlag, Sept. 1999.

[4] J.-M. Couvreur, S. Grivet, and D. Poitrenaud. Designing a LTL model-checker based on unfolding graphs. In Proc. of the ICATPN'00, volume 1825 of LNCS. Springer-Verlag, June 2000.

[5] J.-M. Couvreur, A. Duret-Lutz, and D. Poitrenaud. On-the-fly emptiness checks for generalized Büchi automata. In Proc. of SPIN'05, volume 3639 of LNCS, pages 143-158. Springer-Verlag, Aug. 2005.

[6] A. Duret-Lutz and D. Poitrenaud. SPOT: an extensible model checking library using transition-based generalized Büchi automata. In Proc. MASCOTS'04, pages 76-83. IEEE Computer Society Press, Oct. 2004.

[7] J. Geldenhuys and A. Valmari. More efficient on-thefly LTL verification with Tarjan's algorithm. Theoretical Computer Science, 345(1):60-82, Nov. 2005.

[8] S. Haddad, J.-M. Ilié, and K. Ajami. A model checking method for partially symmetric systems. In Proc. of FORTE/PSTV'O0, volume 183 of IFIP Conference Proceedings. Kluwer, Oct. 2000.

[9] S. Haddad, J.-M. Ilié, and K. Klai. Design and evaluation of a symbolic and abstraction-based model checker. In Proc. of ATVA'04, volume 3299 of LNCS, pages 198-210. Springer-Verlag, Oct. 2004.

[10] J. Hugues, Y. Thierry-Mieg, F. Kordon, L. Pautet, $\mathrm{S}$. Barrir, and T. Vergnaud. On the formal verification of middleware behavioral properties. In Proc. of FMICS'04, volume 133 of ENTCS, pages 139-157. Elsevier, Sept. 2004.

[11] S. Schwoon and J. Esparza. A note on on-the-fly verification algorithms. In Proc. of TACAS'05, volume 3440 of LNCS. Springer-Verlag, Apr. 2005.

[12] H. Tauriainen. A note on the worst-case memory requirements of generalized nested depth-first search. Research Report A96, Helsinki University of Technology, Laboratory for Theoretical Computer Science, Espoo, Finland, Sept. 2005.

[13] Y. Thierry-Mieg, J.-M. Ilié, and D. Poitrenaud. A symbolic symbolic state space representation. In Proc. of FORTE'04, volume 3235 of LNCS, Sept. 2004.

[14] A. Valmari. The state explosion problem. In Lectures on Petri Nets 1: Basic Models, volume 1491 of LNCS, pages 429-528. Springer-Verlag, 1998.

[15] M. Y. Vardi. An automata-theoretic approach to linear temporal logic. In Proc. of Banff'94, volume 1043 of LNCS, pages 238-266. Springer-Verlag, 1996. 